\title{
Does Progress Testing Violate the Principles of Constructive Alignment?
}

\author{
Marcus A. Henning ${ }^{1} \cdot$ Ralph Pinnock ${ }^{2} \cdot$ Craig S. Webster $^{3}$
}

Published online: 16 August 2017

(C) International Association of Medical Science Educators 2017

\section{Introduction}

In the following commentary paper, we consider the possibility of theoretical inconsistencies between one of the most celebrated and well-established theories in higher education, namely constructive alignment, and an increasingly popular approach to assessment, that of progress testing $[1,2]$. Our objective is to consider potential contradictions in the theoretical presumptions underlying each approach with a view to improving our understanding of these presumptions and ultimately the consistency of curricular design and delivery.

\section{Constructive Alignment}

Constructive alignment is an organizing principle used to ensure that each teaching and learning activity in a course corresponds to a specific element of assessment, and in turn, a

Marcus A. Henning

m.henning@auckland.ac.nz

Ralph Pinnock

ralph.pinnock@otago.ac.nz

Craig S. Webster

c.webster@auckland.ac.nz

1 Centre for Medical and Health Sciences Education, University of Auckland, Private Bag 92019, Auckland 1142, New Zealand

2 Dean's Department, Dunedin School of Medicine, University of Otago, P.O. Box 56, 201 Great King Street, Dunedin 9016, New Zealand

3 Centre for Medical and Health Sciences Education and Department of Anaesthesiology, University of Auckland, Private Bag 92019, Auckland 1142, New Zealand specific intended learning outcome. The idea of constructive alignment emerged from the fusing of large theoretical and empirical bodies of work from constructivist learning theory and instructional design $[2,3]$. Constructivist learning theory implies that learners develop understanding and derive meaning through active engagement with their own experiences and those of others, thus leading to the construction of new knowledge. Instructional design contains the notion of alignment by suggesting that instructional situations need to be linked with appropriate learning opportunities and activities. Instructional design coordinates the learning that is determined by the curriculum, through establishing learning objectives that are explicitly stated and matched with appropriate teaching and learning activities [3]. Misalignment occurs if there is a break in this chain such that students may be taught material or engage in learning activities which are not assessed, or when students are assessed on material which they have not been taught.

Further developments based on the concept of constructive alignment are modern approaches to teaching and course development such as the outcomes-based approach and blueprinting. Using the outcomes-based approach, an educator begins by defining the intended learning outcomes of the course or teaching session and then works backwards to develop the teaching and learning activities required to achieve those outcomes $[4,5]$. Elements of assessment are linked to teaching and learning activities, and learning outcomes, and the course is appraised using an evaluation system [4]. Blueprinting is a process of checking and developing the elements of a course or curriculum for comprehensiveness and alignment by completing a matrix encompassing all the components [6]. Constructive alignment is now a fundamental and influential idea in higher education and underpins any discussion about good teaching. Empirical evidence suggests that when alignment occurs in this way, student achievement is 
significantly enhanced in comparison to non-aligned educational systems $[2,3]$.

\section{Progress Testing}

Progress testing has been described as an assessment process whereby students are examined in each year of their study using examination questions designed to represent the level of knowledge of the final qualifying examination [7-10]. Figure 1 shows a conceptual and schematic representation of the process of progress testing. According to this schema, expected performance increases over time. Each successive examination represents a point at which students are asked to answer a subset of test items randomly selected from the same large bank of questions, many of which will be associated with material that students have yet to learn. As students develop their knowledge and skill base, their performance on each examination is expected to increase. The error bars in Fig. 1 propose the theoretical assumption that the "true" assessment of student capability becomes more defined, with less variance, as students advance in their course of study.

Albanese and Case [11] state the defining characteristics of progress testing as:

1. Assessment of student learning based upon "endobjectives" [1] of the curriculum or "competencies that students were expected to demonstrate upon graduation" [12] and is therefore decoupled from the specifics of what students have learned or are learning at any given time.

2. Tests are created to be comprehensive which make them virtually impossible to study for using rote memorization approaches.

3. Scores on individual test administrations are used for formative assessment, not for summative assessment.

4. Whether students are making adequate progress (medium- and high-stakes decisions) is judged on the basis of accumulated performance over several tests to reduce student concern over performance on any single test.

A proposed theoretical advantage of progress testing (stemming from characteristics 1 and 2 above) is that it eliminates the possibility of cramming as students are unable to prepare for the wide range of items on any particular test, because up until the final examination, the questions will be assessing material which has yet to be taught $[13,14]$. For example, at the first annual examination, perhaps only $20 \%$ of questions will be familiar to students as this is the proportion of the curriculum that has been covered, and so even the best students, who have not had previous medical training, should hope to score is a maximum of $20 \%$ correct. This is presumed to "decouple" examinations from student learning, by changing the emphasis from studying for the exam to an accumulation of knowledge over time employing deeper learning processes $[11,13]$. Progress tests are also likely to provoke ways of dealing with uncertainty and promote self-monitoring skills [15]. In addition, statistical methods are being developed to factor in how levels of certainty may impact on scoring the probability of a correct response [15].

When used as intended in a formative way (characteristics 3 and 4 above), progress testing appears to be an efficient and effective method for evaluating gain of knowledge amongst medical students [16]. Progress tests appear to remove the high-stakes nature associated with traditional examinations and to reduce the stress of those being examined [8, 9, 13, $17,18]$. There is some evidence to suggest that progress tests, in comparison to traditional examinations, engender a more meaning-oriented study strategy rather than strategies based on a rote learning approach, although the evidence that it cultivates or motivates learning via more intrinsic or deeplearning mechanisms remains unclear $[8,10,18]$. There is also a belief that progress tests are more suited to students who work consistently and who are in medical schools that promote timely clinical contact [19].
Fig. 1 The conceptual levels of performance for each progress test over the course of an educational program for the first progress test (exam 1) to the final exam at the end of medical training. The asterisk represents the mean score and the graphic indicates uneven variation (as indicated by the error bars) around the conceptual mean score at each exam point

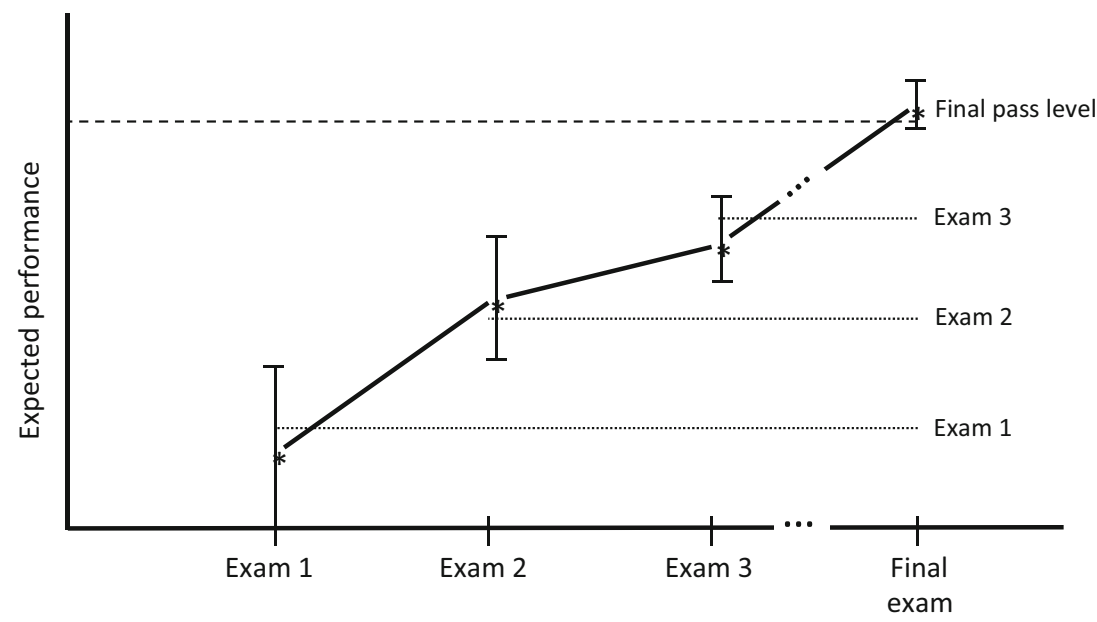




\section{Constructive Alignment and the Practice of Progress Testing}

The primary inconsistency between the theory of constructive alignment and the practice of progress testing is that up until the final examination, students are being tested on material which they have yet to be taught. At best, intermediary tests can be considered to be only partially constructively aligned with the intended learning outcomeswith full alignment occurring only in the final examination, as by this time, teaching and learning activities will have covered the full curriculum (Fig. 1). Advocates of progress testing present this deliberate misalignment as an advantage - they claim this is the decoupling between teaching and assessment activities which encourages deep learning. Furthermore, individual progress tests are intended to be assessments for learning rather than assessments of learning - in the sense that it is purported that testing can promote the element of curiosity and motivation for further learning, which will likely extend beyond the taught curriculum $[8,20]$. However, at least to date, evidence of deep learning engendered by progress testing remains equivocal [18] and almost entirely theoretical.

Scores on individual progress tests are intended to comprise formative assessment only, with medium- and highstakes decisions being made only on consideration of multiple test results. Progress testing appears to be well suited to a formative process, in which feedback is the key to stimulating learning. Konopasek and colleagues [21] have made a powerful and systematic argument that promotes mastery learning that can be associated with progress testing rather than performance learning. In reference to mastery learning, feedback is seen as key to improving one's competence. Consistent with the principle of formative assessment, students will likely learn from uncertainty and seek advice and counsel if they do not know the answer to equivocal questions. In their paper, Konopasek suggests that "virtually," all assessments can be formative in nature, although the way in which results are disseminated requires institutional backing of a formatively focused assessment system. Progress testing as a feedback and formative system appears to advocate the idea of constructive alignment, given that assessment is not only about passing a test but also about delivering feedback that can increase the efficiency and substantive nature of the knowledge acquisition process. This is consistent with other work which found that scaffolding and mentoring were important when delivering formative feedback and this is especially pertinent to students in their early years of training [22].

A further initiative being applied by McMaster University is to ensure that no student passes or fails a year of study based solely on their progress test scores, and that other additional sources of assessment are required for high-stakes decisions
$[11,23]$. However, other universities do not take such a cautious approach in their high-stakes decision-making. The use of multiple progress test scores as a summative assessment during high-stakes decision-making leads to a further potential problem in that each test may make up a significant part of the overall assessment score determining whether a student should continue in their studies. The question then arises of how many progress test results are needed to ensure equitable high-stakes decision-making.

There are a number of potential sources of variance in progress test scores. The performance of individual students in successive tests may vary and may not conform to the expected pattern of growth over time. For example, students may do well in one test and then in the next test do poorly compared with their peers, potentially doing worse than they did in the previous test. Such students may become demoralized and be at risk of greater test anxiety and lowered self-efficacy [14]. This is of particular concern in courses of study such as Medicine, which are typically taken by very competent, highly competitive students, with a tendency to view every examination as summative. The makeup of each progress test may also be variable, even when using a structured blueprint to guide question type, since questions are drawn from a large bank of test items using random selection-hence impacting on the true value of the test in assessing student ability. These sources of variance are particularly important in the early years when the percentage of the items on the test that students will have knowledge of is low (Fig. 1) [24]. Interestingly, very little research has presented variance data from the results of the progress testing. In one study [8], the results of year 2 and 4 progress tests were depicted showing, contrary to what might be ideally expected (Fig. 1), that the variance was greater in year 4 than in year 2. Nonetheless, in this case, this cohort of year 4 students were in their first formal year of progress testing and thus were more exposed to traditional testing in their earlier year of training.

In a further study, Ricketts and Moyeed [25] state that "when progress tests are used to make passfail decisions on individual students, those responsible for assessment should do all they can to improve the accuracy and precision of score estimates." They have presented a systematic method for dealing with the problem of fluctuating measurement error. Using a Bayesian approach, they created a mathematical system for weighting tests according to how much error is inherent in the scores, thus improving and smoothing the precision of the score. Finally, Zahra and colleagues [26] have investigated ways to measure how reliability changes over time and their approach can be applied to individuals or small groups of students. Even though this approach appears to be untested in their paper, it allows for measures of reliability to be factored into any decision being made about a student's true level of performance at any given time. 


\section{Final Thoughts}

The question we initially posed was, "Does progress testing violate the principles of constructive alignment?" In our review of the literature and constant deliberation, our answer is both "yes" and "no." In our view, if progress tests are used summatively and no methods are being applied to consider measurement error, reliability over time, and the uncertain nature of the test, then clearly the answer is "yes." Ricketts and Mayeed state that "approaches based upon grade sequences or linear models have disadvantages, and the relative importance of individual tests in the sequence is not obvious to students or faculty members, which leads to student and staff dissatisfaction."

Nonetheless, if progress tests are given appropriate weightings and are judiciously monitored in terms of how accurately they measure a student's true level of competence, then the random use of items can be factored into the assessment score. In addition, if used formatively, progress tests clearly fit with the theoretical tenor of constructive alignment. This is particularly true if the grades given to students are provided alongside detailed and constructive feedback [21, 22]. Consequently, the uncertain nature of the assessment process is actually a part of the teaching and learning process and can easily be built into the learning objectives or outcomes associated with the course of study.

One area of further research would be to measure variance levels in line with years in training. Accordingly, the theoretical position we have created with Fig. 1 could be refined, refuted, or endorsed. In reality, the actual changes in variance are likely to be less prescriptive and thus the use of the methods endorsed by Rickett and Mayeed [25] and Zahra [26] will be useful in determining suitable algorithms for dealing with raw progress test scores and the uncertainty associated with these resultant test score patterns. This is important if we wish to maintain the integrity of the testing process in terms of providing students with accurate reflections of their true levels of competency.

Notes on Contributors Marcus A. Henning is a senior lecturer in the Centre for Medical and Health Sciences Education at the University of Auckland, New Zealand.

Ralph Pinnock is an Associate Professor and Associate Dean Medical Education in the Dean's Department, Dunedin School of Medicine PO Box 56, Dunedin 9054, New Zealand.

Craig Webster is a senior lecturer in the Centre for Medical and Health Sciences Education at the University of Auckland, New Zealand.

\section{Compliance with Ethical Standards}

Conflict of Interest The authors declare that they have no conflict of interest.

\section{References}

1. Van der Vleuten CPM, Verwijnen GM, Wijnen HFW. Fifteen years of experience with progress testing in a problem-based learning curriculum. Med Teach. 1996;18:102-9.

2. Biggs J. Enhancing teaching through constructive alignment. High Educ. 1996;32(3):347-64.

3. Biggs J, Tang C. Teaching for quality learning at university. Maidenhead: McGraw-Hill and Open University Press; 2011.

4. Prideaux D. $\mathrm{ABC}$ of learning and teaching in medicine: curriculum design. BMJ. 2003;326(7383):268.

5. Harden RM, Crosby JR, Davis MH. AMEE Guide No. 14: outcome-based education: part 1 -an introduction to outcomebased education. Med Teach. 1999;21:7-14.

6. Wilkinson TJ, Wade WB, Knock LD. A blueprint to assess professionalism: results of a systematic review. Acad Med. 2009;84:551-8.

7. Henning M, Chen Y. Medical students and their learning environment: implications for assessments and academic performance. In: Gallagher $\mathrm{CH}$, editor. Academic performance: student expectations, environmental factors and impacts on health. New York: Nova Publisher; 2015. p. 107-22.

8. Lillis S, Yielder J, Mogol V, et al. Progress testing for medical students at the University of Auckland: results from the first year of assessments. J Med Educ Curricular Dev. 2014;2014(1):41-5.

9. Schuwirth LWT, van der Vleuten CPM. The use of progress testing. Perspect Med Educ. 2012;1(1):24-30.

10. Van Berkel HJ, Nuy HJ, Geerligs T. The influence of progress tests and block tests on study behaviour. Instr Sci. 1994;22(4):317-33.

11. Albanese M, Case SM. Progress testing: critical analysis and suggested practices. Adv Health Sci Educ. 2016;21(1):221-34.

12. Arnold L, Willoughby TL. The quarterly profile examination. Acad Med. 1990;65:515-6.

13. Van der Vleuten CPM, Schuwirth LWT, Driessen EW, et al. A model for programmatic assessment fit for purpose. Med Teach. 2012;34(3):205-14.

14. McHarg J, Bradley P, Chamberlain S, Ricketts C, Searle J, McLachlan JC. Assessment of progress tests. Med Educ. 2005;39(2):221-7.

15. Tweed M, Purdie G, Wilkinson T. Low performing students have insightfulness when they reflect-in-action. Med Educ. 2017;51(3): 316-23.

16. Tomic ER, Martins MA, Lotufo PA, Benseñor IM. Progress testing: evaluation of four years of application in the school of medicine. Univ São Paulo Clin. 2005;60(5):389-96.

17. Van der Vleuten CPM, Schuwirth LWT, Muijtjens AMM, Thoben A, Cohen-Schotanus J, Van Boven CPA. Cross institutional collaboration in assessment: a case on progress testing. Med Teach. 2004;26(8):719-25.

18. Chen Y, Henning M, Yielder J, Jones R, Wearn A, Weller J. Progress testing in the medical curriculum: students' approaches to learning and perceived stress BMC Medical Education. 2015;15(147):1-8.

19. Wade L, Harrison C, Hollands J, Mattick K, Ricketts C, Wass V. Student perceptions of the progress test in two settings and the implications for test deployment. Adv Health Sci Educ. 2012;17(4):573-83.

20. Pugh D, Regehr G. Taking the sting out of assessment: is there a role for progress testing? Med Educ. 2016;50:721-9.

21. Konopasek L, Norcini J, Krupat E. Focusing on the formative: building an assessment system aimed at student growth and development. Acad Med. 2016;91(11):1492-7.

22. Harrison CJ, Könings KD, Dannefer EF, Schuwirth LWT, Wass V, van der Vleuten CPM. Factors influencing students' receptivity to formative feedback emerging from different assessment cultures. Perspect Med Educ. 2016;5(5):276-84. 
23. Blake JM, Norman GR, Smith EKM. Report card from McMaster: student evaluation at a problem-based medical school. Lancet. 1995;345(8954):899-902.

24. Ravesloot CJ, Van der Schaaf MF, Muijtjens AMM, et al. The don't know option in progress testing. Adv in Health Sci Educ. 2015;20: $1325-38$.
25. Ricketts C, Moyeed R. Improving progress test score estimation using Bayesian statistics. Med Educ. 2011;45(6):570-7.

26. Zahra D, Hedge C, Pesola F, Burr S. Accounting for test reliability in student progression: the reliable change index. Med Educ. 2016;50(7):738-45. 